\title{
Compliance Analysis of an Under-Actuated Robotic Finger
}

\author{
Martin Wassink, Raffaella Carloni and Stefano Stramigioli
}

\begin{abstract}
Under-actuated robotic hands have multiple applications fields, like prosthetics and service robots. They are interesting for their versatility, simple control and minimal component usage. However, when external forces are applied on the finger-tip, the mechanical structure of the finger might not be able to resist them. In particular, only a subset of disturbance forces will meet finite compliance, while forces in other directions impose null-space motions (infinite compliance).

Motivated by the observation that infinite compliance (i.e. zero stiffness) can occur due to under-actuation, this paper presents a geometric analysis of the finger-tip compliance of an under-actuated robotic finger. The analysis also provides an evaluation of the finger design, which determines the set of disturbances that is resisted by finite compliance.

The analysis relies on the definition of proper metrics for the joint-configuration space. Trivially, without damping, the mass matrix is used as a metric. However, in the case of damping (power losses), the physical meaningful metric to be used is found to be the damping matrix.
\end{abstract}

Simulation experiments confirm the theoretical results.

\section{INTRODUCTION}

A new generation of full-service robots is being developed for the domestic appliances market. Industry shifts towards automated production of customized, small batch and short life-cycle products [1]. And, also in prosthetics major breakthroughs are coming out [2]. In all of these application fields, versatile robots are needed to execute a large range of varying tasks in unstructured environments.

Many of these prospected tasks require to interact in unstructured human environments and to deal with unknown objects. A versatile end-effector, alike the human hand, is one of the critical components for successfully developing this new generation of robotic devices. Hence, dexterous robotic hands that have human hand functionality and dimensions are believed to be the required end-effectors. These dexterous robotic hands should be able to grasp (ir)regular objects (pinch and enveloping grasps) and to manipulate objects and fingers (e.g. pre-shaping). Compromises on dimensions, weights, complexity, reliability, functionality and costs complicate the development of such robotic hands.

A novel minimal component biomimetic robotic finger concept with variable compliance was introduced to alter these complications [3]. The concept utilizes under-actuation (inspired by [4] and [5]), which reduces both the number

This work has been carried out as part of the FALCON project under the responsibility of the Embedded Systems Institute with Vanderlande Industries as the industrial partner. This project is partially supported by the Netherlands Ministry of Economic Affairs under the Embedded Systems Institute (BSIK03021) program.

\{m.wassink, r.carloni, s.stramigioli\}@utwente.nl, Control Engineering, Faculty of Electrical Engineering, Mathematics \& Computer Science, University of Twente, The Netherlands. of heavy power actuators and the grasp control complexity, while it improves versatility. Variable mechanical compliance is added by antagonistic non-linear spring elements in the driving tendons to further enhance task adaptability and grasp robustness. Joint locks are used to restore full manipulability for e.g. pre-shaping and gesturing. This human-inspired concept is currently under investigation for versatile applications, such as prosthetic hands.

The under-actuated driving mechanism results in a singular transmission between the series elastic non-linear springs in the tendons and the joints to be actuated. These singularities complicate the compliance analysis of under-actuated fingers. Nevertheless, thorough understanding of these properties is crucial to utilize compliance in enhancing grasp robustness. This paper aims to present compliance properties of underactuated robotic fingers and in particular the variable compliance properties of the novel under-actuated robotic finger.

The paper is organized as follows. Section II summarizes the model of the under-actuated finger presented in [3]. Section III analyzes the compliance properties of the underactuated finger. Then, Section IV complements the analysis with a discussion on the choice of metrics and Section V adds some design considerations. Presented theory is validated with simulation experiments in Section VI. The paper finishes with conclusions and future work.

\section{Finger Model for Compliance AnAlysis}

Fig. 1 presents the model of the variable compliance under-actuated robotic finger under investigation. The model variables, as presented in Fig. 1 and used throughout the paper, are listed below:

q: $\quad q \in \mathcal{Q} \subset \mathbb{R}^{3}$ is the finger configuration (jointangles) on the configuration manifold $\mathcal{Q}$.

$\dot{q}: \quad \dot{q} \in T_{q} \mathcal{Q}$ is the time derivative of $q$, being elements (vectors) of the tangent space of $\mathcal{Q}$ at $q$.

$\tau: \quad \tau \in T_{q}^{*} \mathcal{Q}$ are torques on the joints, being elements (co-vectors) of the co-tangent space of $\mathcal{Q}$ at $q$.

$s: \quad s \in \mathcal{S} \subset \mathbb{R}^{2}$ are the positions of the tendon.

$\dot{s}: \quad \dot{s} \in T_{s} \mathcal{S}$ are the time derivatives of $s$, being elements (vectors) of the tangent space of $\mathcal{S}$ at $s$.

$F_{s}: \quad F_{s} \in T_{s}^{*} \mathcal{S}$ are the tendon forces, being elements (co-vectors) of the co-tangent space $T_{s}^{*} \mathcal{S}$ at $s$.

$\ell: \quad \ell \in \mathcal{L} \subset \mathbb{R}^{2}$ are the elongations of the non-linear elastic elements.

$\dot{\ell}: \quad \dot{\ell} \in T_{\ell} \mathcal{L}$ are the time derivatives of $\ell$, being elements (vectors) of the tangent space of $\mathcal{L}$ at $\ell$.

$F_{\ell}: \quad F_{\ell} \in T_{\ell}^{*} \mathcal{L}$ are the elastic forces equal to $F_{s}$.

$W_{e}: \quad W_{e} \in s e^{*}(3)_{H(t)}$ is the externally applied wrench (generalized 6 d.o.f. force) on the finger- 


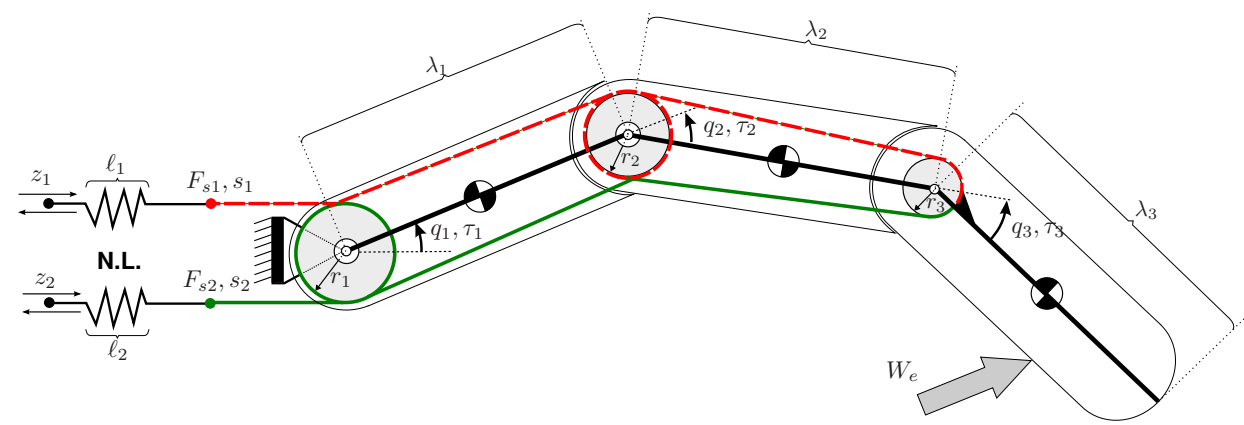

Fig. 1. Model of variable compliance under-actuated robotic finger. Input positions $z$ are controlled by a position controller. Non-linear elastic elements (denoted by N.L. with lengths $\ell_{1}, \ell_{2}$ ) are used in the series elastic antagonistic tendon drives. The tendons are routed about the idle pulleys with radii $r_{1}$ and $r_{2}$ and about the fixed pulley (fixed to distal phalanx, i.e. finger-tip) with radius $r_{3}$. The lengths of the phalanges are captured in $\lambda_{i}, i \in\{1,2,3\}$.

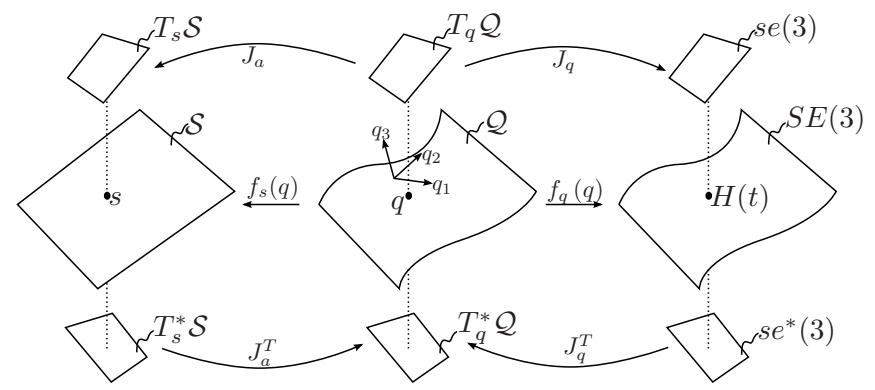

Fig. 2. Model variables; The three joint-angles $q_{1}, q_{2}, q_{3}$ form a natural coordinate base to span the configuration space $\mathcal{Q}$.

tip ( $3^{\text {rd }}$ phalanx). The wrench space $s e^{*}(3)_{H(t)}=$ $T_{H(t)}^{*} S E(3)$ is the co-tangent space of the group of rigid transformations $H(t)$, called $S E(3)$, which denotes the special Euclidian group, at rigid transformation $H(t)$ [6].

T: $\quad T \in \operatorname{se}(3)$ is the twist (generalized 6 d.o.f. rigid body motions) of the finger-tip. The twist space $s e(3)_{H(t)}=T_{H(t)} S E(3)$ is the tangent space of $S E(3)$ at $H(t)$ [6].

$u$ : The inputs $u \in T_{z} \mathcal{Z}$ represent the velocities in the tangent space $T_{z} \mathcal{Z}$ of the tendon actuation position space $\mathcal{Z} \subset \mathbb{R}^{2}$ at $z \in \mathcal{Z}$ (i.e. $u=\dot{z}$ ).

Fig. 2 shows the listed variables as elements of different spaces and their inter-relating mappings. The function $f_{q}$ : $\mathcal{Q} \rightarrow S E(3)$ maps the joint configuration into a rigid body transformation for the finger-tip, while $f_{s}: \mathcal{Q} \rightarrow \mathcal{S}$ maps the joint configuration to the tendon positions. The three jointangles $q_{1}, q_{2}, q_{3}$ span the bases of the configuration space $\mathcal{Q}$, which results in the following equation for $f_{s}(q)$ :

$$
f_{s}(q):\left\{\begin{array}{l}
s_{1}=r_{1} \cdot q_{1}+r_{2} \cdot q_{2}+r_{3} \cdot q_{3} \\
s_{2}=-r_{1} \cdot q_{1}-r_{2} \cdot q_{2}-r_{3} \cdot q_{3}
\end{array} .\right.
$$

The differential mappings (Jacobians) of $f_{q}$ and $f_{s}$ relate the tangent and co-tangent spaces in a dual manner. The geometric Jacobian $J_{q}(q)$, with short notation $J_{q}:=J_{q}(q)$, defines the tangent map and dually the co-tangent map [6]:

$$
\begin{array}{lll}
J_{q} & : T_{q} \mathcal{Q} \rightarrow s e(3) & \left(T=J_{q} \cdot \dot{q}\right) \\
J_{q}^{T}:: s^{*}(3) \rightarrow T_{q}^{*} \mathcal{Q} & \left(\tau^{T}=J_{q}^{T} \cdot W_{e}^{T}\right)
\end{array} .
$$

The actuation Jacobian $J_{a}$ defines the (co-)tangent maps between the finger configuration and tendon position spaces:

$$
\begin{array}{lllc}
J_{a} & : & T_{q} \mathcal{Q} \rightarrow T_{s} \mathcal{S} & \left(\dot{s}=J_{a} \cdot \dot{q}\right) \\
J_{a}^{T} & : & T_{s}^{*} \mathcal{S} \rightarrow T_{q}^{*} \mathcal{Q} & \left(\tau^{T}=J_{a}^{T} \cdot F_{s}^{T}\right)
\end{array} .
$$

\section{Finger-tip Compliance AnAlysis}

The finger-tip compliance matrix $\left(C_{f}\right)$ under investigation defines the infinitesimal finger-tip displacement $\delta T \in s e(3)$ (i.e. infinitesimal deformation twist) of the finger in response to an externally applied infinitesimal wrench $\delta W_{e} \in s e^{*}(3)$ around an equilibrium:

$$
\delta T=C_{f} \cdot \delta W_{e}^{T},
$$

where $\delta T=T \cdot d t=J_{q} \cdot \delta q$ and $\delta q \in T_{q} \mathcal{Q}$ is an infinitesimal joint displacement around an equilibrium configuration [7].

For the compliance analysis, the controller inputs remain constant, i.e. $u=0$. Note that, since the controller inputs $u$ are fixed at position $z$, the tendon positions $s$ are equal to the elongations $\ell$ of the elastic elements. In this case $\dot{s}=\dot{\ell}$, such that from Eq. 3 it follows that $\dot{\ell}=J_{a} \cdot \dot{q}$.

The analysis is divided into three parts. First, the finger-tip compliance problem is solved by decomposing the configuration space into two sub-spaces. Then, it is shown for which wrenches the compliance remains finite. These insights also reveal some design considerations. Finally, a proper choice of metric for the decomposition is discussed.

\section{A. Variable finger-tip compliance}

Joint compliance matrix $C_{q}$ is defined by $\delta q=C_{q}$. $\delta \tau^{T}$, where $\delta \tau \in T_{q}^{*} \mathcal{Q}$ are the infinitesimal joint torques around some equilibrium. Assuming existence of $C_{q}$, premultiplication with $J_{q}$ and substitution of $\delta \tau^{T}=J_{q}^{T} \cdot \delta W_{e}^{T}$ (Eq. 2) leads to $\delta T=J_{q} C_{q} J_{q}^{T} \cdot \delta W_{e}^{T}$, such that

$$
C_{f}=J_{q} C_{q} J_{q}^{T} \text {. }
$$

The tangent mapping $J_{a}$ is non-invertible due to underactuation. Hence, there is no trivial expression for the jointcompliance $C_{q}$ [3]. Alternatively, its inverse, the joint stiffness $K_{q}$, defined through $\delta \tau^{T}=K_{q} \cdot \delta q$, was found to be the pullback of $\frac{\partial^{2} H_{\ell}}{\partial \ell^{2}}(\ell)$ for the map $f_{s}(q)$ [3];

$$
K_{q}=J_{a}^{T} \cdot \frac{\partial^{2} H_{\ell}}{\partial \ell^{2}}(\ell) \cdot J_{a},
$$




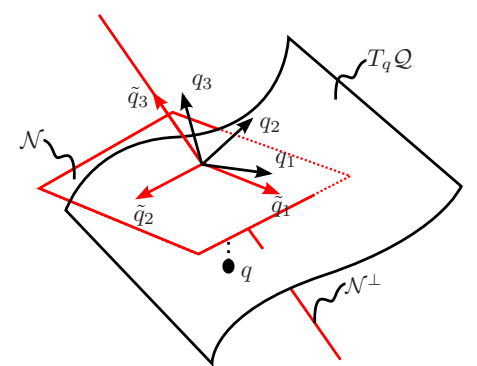

Fig. 3. Visualization of coordinate change by $\operatorname{ker}\left(J_{a}\right) . T_{q} \mathcal{Q}$ is decomposed into $\mathcal{N}$ and $\mathcal{N}^{\perp}$, s.t. $T_{q} \mathcal{Q}=\mathcal{N} \oplus \mathcal{N}^{\perp}$. Subspace $\mathcal{N}$ is the null-space of $J_{a}: \mathcal{N}=\operatorname{ker} J_{a}$, while $\mathcal{N}^{\perp}$ is its reciprocal space.

where

$$
\frac{\partial^{2} H_{\ell}}{\partial \ell^{2}}(\ell)=\left(\begin{array}{cc}
\frac{\partial^{2} H_{\ell}}{\partial \ell_{1}^{2}}\left(\ell_{1}\right) & 0 \\
0 & \frac{\partial^{2} H_{\ell}}{\partial \ell_{2}^{2}}\left(\ell_{2}\right)
\end{array}\right)
$$

and $H_{\ell}$ is the energy storage function of the elastic elements in the driving tendons.

To find an intuitive expression for $\tilde{K}_{q}$ that allows to resolve the finger-tip compliance $C_{q}$, new coordinates $\tilde{q}$ are chosen by defining a coordinate transformation $R$ on $T_{q} \mathcal{Q}$ :

$$
\delta q=R \cdot \delta \tilde{q},
$$

where $\delta q, \delta \tilde{q} \in T_{q} \mathcal{Q}$ are expressed in different coordinates; i.e. the original physical joint angle coordinates $q=\left(q_{1}, q_{2}, q_{3}\right)$ and new coordinates $\tilde{q}=\left(\tilde{q}_{1}, \tilde{q}_{2}, \tilde{q}_{3}\right)$. The columns in $R$ form the new set of base vectors that spans $\mathcal{Q}$ expressed as vectors in the joint angle coordinates $q$. Dually, $R^{-T}$ on $T_{q}^{*} \mathcal{Q}$ gives $\delta \tau=R^{-T} \cdot \delta \tilde{\tau}$.

This coordinate transformation results in a joint stiffness $\tilde{K}_{q}$ in the new coordinates $\tilde{q}$ :

$$
\tilde{K}_{q}=R^{T} \cdot K_{q} \cdot R
$$

such that $\delta \tilde{\tau}^{T}=\tilde{K}_{q} \cdot \delta \tilde{q}$. Hence,

$$
\delta \tau^{T}=R^{-T} \cdot \tilde{K}_{q} \cdot R^{-1} \cdot \delta q .
$$

\section{B. Joint space decomposition}

In order to choose a helpful coordinate transformation, the following understanding is important. Some directions, $\delta q \in$ ker $J_{a}$, project through $J_{a}$ to zero displacement in $\dot{\ell}$, which corresponds to zero stiffness. Other directions $\left(\delta q \notin \operatorname{ker} J_{a}\right.$ ) do impose a change in elongation in the elastic elements, which reflects finite stiffness.

Hence, the mapping $J_{a}$ is used to decompose $T_{q} \mathcal{Q}$ into subspace $\mathcal{N}$ and $\mathcal{N}^{\perp}$, such that $T_{q} \mathcal{Q}=\mathcal{N} \oplus \mathcal{N}^{\perp}$. This is visualized in Fig. 3. Subspace $\mathcal{N}$ is the null-space of $J_{a}$ :

$$
\mathcal{N}=\operatorname{ker} J_{a}=: \operatorname{span}\left(n_{1}, n_{2}\right),
$$

while $\mathcal{N}^{\perp}=\operatorname{span}\left(n^{\perp}\right)$ is its reciprocal space. The vectors $n_{1}, n_{2}, n^{\perp} \in T_{q} \mathcal{Q}$ are expressed in joint coordinates $q$. Reciprocality in $T_{q} \mathcal{Q}$ is defined by the weighted inner product on $T_{q} \mathcal{Q}$ being equal to zero. The weighted inner product on $T_{q} \mathcal{Q}$ is:

$$
u<\bullet>_{M_{q}} w=u^{T} M_{q} w \quad u, w \in T_{q} \mathcal{Q},
$$

where $M_{q}$ is a metric on $T_{q} \mathcal{Q}$. Using this inner product definition, $n^{\perp}$ is found to be:

$$
n^{\perp}=M_{q}^{-1} \cdot \operatorname{Im}\left(J_{a}^{T}\right) .
$$

Thus, $R$ becomes

$$
R=\left(\begin{array}{lll}
n_{1} & n_{2} & n^{\perp}
\end{array}\right)=\left(\begin{array}{lll}
n_{1} & n_{2} & M_{q}^{-1} v
\end{array}\right),
$$

where $v=\operatorname{Im}\left(J_{a}^{T}\right) \in T_{q}^{*} \mathcal{Q}$.

Expressing the infinitesimal finger displacement in the new coordinates $(\delta \tilde{q})$ immediately shows whether there are nullspace motions or reciprocal motions:

$$
\delta \tilde{q}_{\text {null-space }}=\left(\begin{array}{l}
\bullet \\
\bullet \\
0
\end{array}\right) \in \mathcal{N}, \quad \delta \tilde{q}_{\text {reciprocal }}=\left(\begin{array}{l}
0 \\
0 \\
\bullet
\end{array}\right) \in \mathcal{N}^{\perp},
$$

where $\bullet$ represents some non-zero number.

Using the presented coordinate transformation $\mathrm{R}, \tilde{K}_{q}$ is found to be:

$$
\begin{aligned}
\tilde{K}_{q} & =R^{T} \cdot K_{q} \cdot R \\
& =R^{T} \cdot J_{a}^{T} \cdot \frac{\partial^{2} H_{\ell}}{\partial \ell^{2}}(\ell) \cdot J_{a} \cdot R \\
& =\left(\begin{array}{lll}
0 & 0 & 0 \\
0 & 0 & 0 \\
0 & 0 & \beta
\end{array}\right),
\end{aligned}
$$

with

$$
\beta=\left(\frac{\partial^{2} H_{\ell}}{\partial \ell_{1}^{2}}\left(\ell_{1}\right)+\frac{\partial^{2} H_{\ell}}{\partial \ell_{2}^{2}}\left(\ell_{2}\right)\right) \cdot\left(v^{T} M_{q}^{-1} v\right)^{2},
$$

which clearly shows that stiffness is only reflected in the reciprocal directions, which turns out to be $\beta$ (Eq. 13). Whereas, null-space motions experience zero stiffness (Eq. 13), i.e. infinite compliance.

Interestingly, the stiffness is the sum of the parallel linearized stiffnesses of the non-linear elastic elements in the driving tendons multiplied by the square of a weighted transmission. This is recognized as how generally stiffness is reflected through transmissions. The weighting metric used here is the dual metric of the metric $M_{q}$ on $T_{q} \mathcal{Q}$, i.e. $M_{q}^{-1}$ on the space of torques $T_{q}^{*} \mathcal{Q}$ to which $v$ belongs.

As noted, torques in the null-space directions will excite infinite motions. With Eq. 13, the joint stiffness relation (Eq. 10) becomes:

$$
\delta \tau^{T}=R^{-T}\left(\begin{array}{ccc}
0 & 0 & 0 \\
0 & 0 & 0 \\
0 & 0 & \beta
\end{array}\right) R^{-1} \cdot \delta q .
$$

Hence, for the joint compliance, which is inversely related to $K_{q}$, the following is concluded:

$$
C_{q}=\left\{\begin{array}{cl}
R \cdot \frac{1}{\beta} \cdot R^{T} & \forall \delta \tau^{T} \in \operatorname{Im}\left(J_{a}^{T}\right) \\
\infty & \forall \delta \tau^{T} \notin \operatorname{Im}\left(J_{a}^{T}\right)
\end{array},\right.
$$

such that the finger-tip compliance (Eq. 5) becomes

$$
C_{f}=\left\{\begin{array}{cc}
J_{q} R \cdot \frac{1}{\beta} \cdot R^{T} J_{q}^{T} & \forall J_{q}^{T} \delta W_{e}^{T} \in \operatorname{Im}\left(J_{a}^{T}\right) \\
\infty & \forall J_{q}^{T} \delta W_{e}^{T} \notin \operatorname{Im}\left(J_{a}^{T}\right)
\end{array},\right.
$$




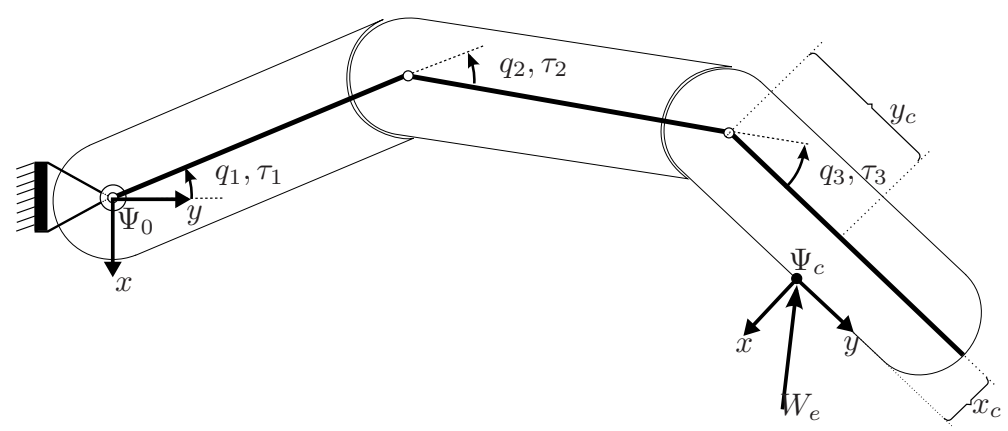

Fig. 4. External wrench $W_{e}$ applied at some contact point on finger-tip. Local coordinates $\Psi_{c}$ are placed at the contact point. $W_{e}$ maps to torques on the joints. This mapping depends on geometric parameters: location of contact point ( $x_{c}$ and $\left.y_{c}\right)$ and lengths of the first two phalanges, $\lambda_{1}$ and $\lambda_{2}$.

which shows that for $J_{q}^{T} \delta W_{e}$ that have elements in the nullspace of $J_{a}$, infinite twists $\delta T$ will be induced, implying infinite compliance. Hence only finite compliance exists for a limited set of wrenches $W_{c}$ :

$$
W_{c}=\left\{\delta W_{e} \in s e^{*}(3) \mid J_{q}^{T} \delta W_{e}^{T} \in \operatorname{Im}\left(J_{a}^{T}\right)\right\} .
$$

Eq. 14 and Eq. 16 confirm that if the elastic elements are non-linear, then the finger-tip compliance can be altered by changing their lengths $\ell$.

\section{Physically Consistent Metric $\left(M_{q}\right)$}

The previous section presented the finger-tip compliance analysis. The specific choice of coordinate transformation $R$ on $T_{q} \mathcal{Q}$ allows to decompose the joint space $\mathcal{Q}$ to describe the finger-tip compliance $C_{f}$ in Eq. 16. The new coordinates $\tilde{q}$, span by base vectors $n_{1}, n_{2}$ and $n^{\perp}$, split the space $T_{q} \mathcal{Q}$ into two parts, based on the kernel of the tangent mapping $J_{a}(\mathcal{N})$ and its reciprocal space $\left(\mathcal{N}^{\perp}\right)$, see Fig. 3.

The expression for $C_{f}$ is given in Eq. 16, which depends on the choice of metric $M_{q}$. Hence, the calculated value of the compliance does change for different metrics. Of course, in reality, only one compliance value exists. Thus, to find physically meaningful compliance values, the correct metric must be used. The correct metric defines a physically meaningful measure for which nature tries to minimize, as discussed in [8], [9].

In robotics, the dynamics are often described by (ignoring gravity) $M(q) \ddot{q}+C(q, \dot{q}) \dot{q}=\tau$, where $M(q)$ is the mass matrix and $C(q, \dot{q})$ the Coriolis matrix and a damping term is left out of the equations. For these systems, it turns out to be trivial to use $M(q)$ as the physically meaningful metric on $T_{q} \mathcal{Q}$, since $\dot{q}^{T} M(q) \dot{q}$ represents kinetic energy [9]. However, it is not always trivial to find such a metric [9]. For the case in which damping is modeled in the mechanism dynamics, no metric was found in literature.

For the robotic finger, it was observed in simulation (as modeled in [3]), that the metric $M_{q}$ to be used appears to be different for two cases: without and with damping on the joints. Damping torques $\tau_{b}$ on the joints are modeled by:

$$
\tau_{b}=\left(\begin{array}{ccc}
b_{1} & 0 & 0 \\
0 & b_{2} & 0 \\
0 & 0 & b_{3}
\end{array}\right) \cdot \dot{q}=B \cdot \dot{q} .
$$

The metric to be used is discussed for both cases:

1) Without damping: any external wrench $\delta W_{e} \in W_{c}$ induces vibrations in the join motions $q$ around an infinitesimal displacement of the equilibrium configuration $\delta q_{e}$. In this case, $\delta q_{e}$ is analytically determined by $\delta q_{e}=C_{q} \cdot J_{q}^{T} \cdot \delta W_{e}^{T}$, with $C_{q}$ described in Eq. 15 and the metric $M_{q}=M(q)$, the mass matrix of the finger dynamics. This coincides with [9].

2) With damping: any external wrench $\delta W_{e} \in W_{c}$ induces a steady state infinitesimal displacement of the equilibrium configuration $\delta q_{e}$. In this case, $\delta q_{e}$ is analytically determined by $\delta q_{e}=C_{q} \cdot J_{q}^{T} \cdot \delta W_{e}^{T}$, with $C_{q}$ described in Eq. 15 and the metric $M_{q}=B$, the damping matrix of the finger dynamics (Eq. 18).

Note that damping $B$ is a physically meaningful metric on $T_{q} \mathcal{Q}$, since $\dot{q}^{T} B \dot{q}$ represents power loss due to damping in the joints. Power losses are minimized by nature.

\section{Under-ACtuated Finger Design Considerations}

Besides an expression for the finger-tip compliance, Section III also indicated that finite compliance is only reflected against a limited set of finger-tip wrenches, i.e. $\delta W_{c}$ (Eq. 17). This section investigates which wrenches actually admit $\delta W_{e} \in W_{c}$ and presents derived design considerations.

\section{A. Finite Compliance Wrenches: $\delta W_{c}$}

Fig. 4 shows the kinematics of the situation under investigation. An external wrench $W_{e}$ is applied at some contact point on the finger-tip, parameterized by distances $x_{c}$ and $y_{c}$. Expressing $W_{e}$ in local coordinates $\Psi_{c}$, gives: ${ }^{c} W_{e}=\left(\begin{array}{llllll}\tau_{x} & \tau_{y} & \tau_{z} & f_{x} & f_{y} & f_{z}\end{array}\right)$, where the first three elements are moments about the coordinate axis of $\Psi_{c}$ and the remaining three elements represent a force, expressed as vector in $\Psi_{c}$. All non-zero moments and forces in $W_{e}$ are the moments and forces that are transmitted through the contact and will impose torques on the joints.

To find an expression for the joint torques, the coordinates of $W_{e}$ are changed to those in which the Jacobian mapping $\left(J_{q}^{T}\right)$ is expressed, e.g. the fixed world coordinate frame $\Psi_{0}$, by applying the adjoint mapping [6]:

$$
{ }^{0} W_{e}^{T}=\left(a d_{H_{0}^{c}}\right)^{T}{ }^{c} W_{e}^{T} .
$$


With Eq. 2, the torques on the joints as a result of $W_{e}$ at a contact point $\left(x_{c}, y_{c}\right)$ on the finger-tip are found to be:

$$
\begin{aligned}
\tau_{e} & =J_{q}^{T} \delta W_{e} \\
& =\left(\begin{array}{c}
f_{y} s q_{3} \lambda_{2}-f_{x} c q_{3} \lambda_{2}+f_{y} c q_{3} s q_{2} \lambda_{1}-f_{x} y_{c} \\
-f_{x} c q_{3} c q_{2} \lambda_{1}+f_{x} s q_{3} s q_{2} \lambda_{1} \\
+f_{y} s q_{3} c q_{2} \lambda_{1}+f_{y} x_{c}+\tau_{z} \\
f_{y} s q_{3} \lambda_{2}-f_{x} c q_{3} \lambda_{2}+\tau_{z}-f_{x} y_{c}+f_{y} x_{c} \\
\tau_{z}-f_{x} y_{c}+f_{y} x_{c}
\end{array}\right) .
\end{aligned}
$$

Hence, to have $\delta W_{e} \in W_{c}$ for a certain configuration $q$, it must hold that $\tau_{e} \in \operatorname{Im}\left(J_{a}^{T}\right)$.

\section{B. Finger Design Trade-off}

For the under-actuated finger under consideration, shown in Fig. 1, this implies that the following design trade-off equality must hold:

$$
a \cdot\left(\begin{array}{c}
r_{1} \\
r_{2} \\
r_{3}
\end{array}\right)=\left(\begin{array}{c}
f_{y} s q_{3} \lambda_{2}-f_{x} c q_{3} \lambda_{2}+f_{y} c q_{3} s q_{2} \lambda_{1}-f_{x} y_{c} \\
-f_{x} c q_{3} c q_{2} \lambda_{1}+f_{x} s q_{3} s q_{2} \lambda_{1} \\
+f_{y} s q_{3} c q_{2} \lambda_{1}+f_{y} x_{c}+\tau_{z} \\
f_{y} s q_{3} \lambda_{2}-f_{x} c q_{3} \lambda_{2}+\tau_{z}-f_{x} y_{c}+f_{y} x_{c} \\
\tau_{z}-f_{x} y_{c}+f_{y} x_{c}
\end{array}\right),
$$

with $a \in \mathbb{R}$ some scalar multiplier, i.e. actuation force.

Hence, the design parameters $\lambda_{i}$ (phalanx lengths) and $r_{i}$ (pulley radii) together with the contact point $\left(x_{c}, y_{c}\right)$ and the applied contact forces $\left(f_{x}, f_{y}, \tau_{z}\right)$ all together determine whether the applied force meets finite compliance. Note that these forces are also forces that can be transmitted from the actuators to the contact point. Clearly, designing the robotic finger for a specific robotic hand involves considering which forces (in which configurations) have to be generated and need to be resisted with finite compliance.

As an example: suppose that for the targeted robotic grasping task it is required to compliantly resist a external force along the $x$ axis of $\Psi_{0}$ (i.e. ${ }^{c} W_{e}=\left(0,0,0, f_{x}, 0,0\right)$ at the finger tip ( $\left.y_{c}=\lambda_{3}, x_{c}=0\right)$ in a straight finger configuration $(q=0)$. Then the design trade-off equality, Eq. 20, becomes:

$$
n \cdot\left(\begin{array}{l}
r_{1} \\
r_{2} \\
r_{3}
\end{array}\right)=\left(\begin{array}{c}
-\left(\lambda_{2}+\lambda_{3}+\lambda_{1}\right) \\
-\left(\lambda_{2}+\lambda_{3}\right) \\
-\lambda_{3}
\end{array}\right) \cdot f_{x},
$$

which shows that the design must hold:

$$
\frac{r_{1}}{\lambda_{2}+\lambda_{3}+\lambda_{1}}=\frac{r_{2}}{\lambda_{2}+\lambda_{3}}=\frac{r_{3}}{\lambda_{3}} .
$$

This general design analysis coincides with the equilibrium point observations in [5]. After the design is fixed, in other configurations, other contact points on the finger-tip and other external wrenches are necessary to admit $\delta W_{e} \in W_{c}$.

\section{VALIDATION By SimUlation}

\section{A. Method}

The theoretical results of the previous section are proved by simulation experiments. A dynamic model of the underactuated finger as sketched in Fig. 1 and 4 was created with

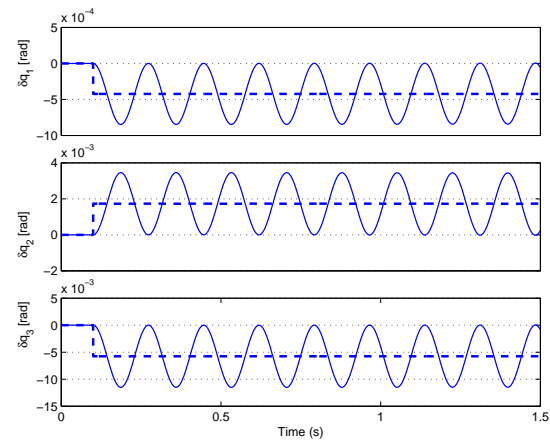

Fig. 5. Case-1: No damping. Infinitesimal joint displacement $\delta q$ due to external disturbance $\delta W_{e}$ : simulated response [solid line] vs. analytically determined response [dashed line], using metric: $M_{q}=M(q)$.

the port-based simulation package 20 -sim ${ }^{1}$. The following design parameters were chosen: $\lambda_{1}=\lambda_{2}=\lambda_{3}=0.04$ $m, r_{1}=0.01, r_{2}=0.00666, r_{3}=0.00333 \mathrm{~m}$. For the experiments, the non-linear elastic elements were simulated with two linear springs with stiffnesses $k_{1}=100 \mathrm{~N} / \mathrm{m}$, $k_{2}=10,000 \mathrm{~N} / \mathrm{m}$.

In the simulation experiments an external infinitesimal wrench $\delta^{c} W_{e}=(0,0,0,0.01,0,0) N$ is applied at $x_{c}=$ $0, y_{c}=\lambda_{3}=0.04 \mathrm{~m}$ on a straight finger configuration $q=0$ rad., such that the design trade-off equality from the example, Eq. 21, is satisfied. The external force $\delta^{c} W_{e}$ is applied as step-function, induced at $t=0.1 \mathrm{~s}$.

The goal of the simulation experiments was to investigate the infinitesimal equilibrium displacement $\delta q_{e}$ after applying $\delta^{c} W_{e}$ for different values of damping $\left(b_{1}, b_{2}, b_{3}\right)$, phalanx masses $\left(m_{1}, m_{2}, m_{3}\right)$ and phalanx moments of inertia about the out-of-plane axis in the center of mass of each phalanx $\left(I_{z 1}, I_{z 2}, I_{z 3}\right)$. For each test-set is was verified whether the experimentally determined $\delta q_{e}$ could be analytically explained by using the metric $M_{q}=M(q)$ or $M_{q}=B$.

Some representative simulation experiments are discussed. For this set of results, the masses of the phalanges were chosen to be $m_{1}=0.1, m_{2}=0.4, m_{3}=0.2 \mathrm{~kg}$ and the moments of inertia $I_{z 1}=1 e^{-5}, I_{z 2}=4 e^{-5}, I_{z 3}=$ $2 e^{-5} \mathrm{kgm}^{2}$. Representative means that equal results were obtained for other parameter values and mass distributions. Two distinct cases are investigated: no damping (case-1)and with damping (case-2). Also varying compliance with nonlinear elastic elements is investigated.

\section{B. Results}

1) No-damping: Fig. 5 shows the response of the infinitesimal joint displacement for the case without damping in the joints. The plot shows that the vibrations are exactly symmetrically around the analytically determined $\delta q_{e}$, which confirms that the metric to be used should be the mass matrix of the finger dynamics $M(q)$.

2) Damping: Fig. 6 shows the response of the infinitesimal joint displacement for the case with damping in the

\footnotetext{
${ }^{1}$ see http://www.20sim.com
} 


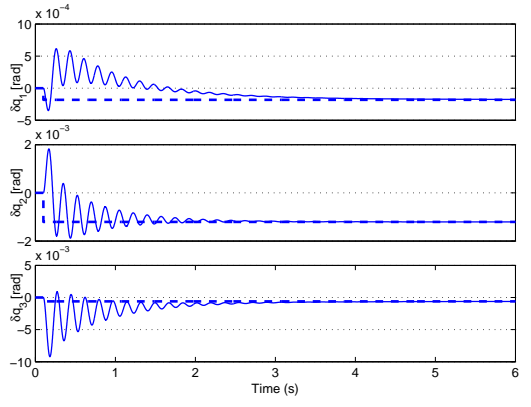

Fig. 6. Case-2: Damping $\left(b_{1}=0.001, b_{2}=0.0001, b_{3}=0.0001\right.$ $\mathrm{Ns} / \mathrm{m}$ ). Infinitesimal joint displacement $\delta q$ : simulated response [solid line] vs. analytically determined response [dashed line], using metric: $M_{q}=B$.

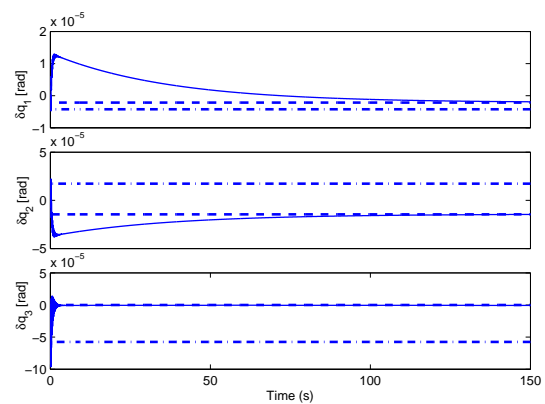

Fig. 7. Case-2: Little Damping $\left(b_{1}=1 e^{-5}, b_{2}=1 e^{-6}, b_{3}=1 e^{-4}\right.$ $\left.N s / m, \delta^{c} W_{e}=(0,0,0,0.0001 \quad N, 0,0)\right)$. Infinitesimal joint displacement $\delta q$ : simulated [solid line] vs. analytically determined response, using metric: $M_{q}=B$ [dashed line] and $M_{q}=M(q)$ [dashed-dotted line].

joints. The plot shows that the simulated joint displacements exactly converge to the the analytically determined $\delta q$. This confirms that the metric to be used, for this case, should be the joint damping matrix of the finger $B$.

Also Fig. 7 confirms that even for small damping values the metric to be used must be $B$. The figure also shows that the mass matrix $M(q)$ as a metric gives incorrect results.

Both cases also confirm that the design trade-off equality from the example Eq. 21, is satisfied for the applied wrench, such that equilibrium is truly reached.

\section{Variable Compliance}

The results so far have shown the existence of finite compliance and confirmed the analytically determined compliance. It was also experimentally tested and verified in simulation that the compliance can be varied by changing the input position $z$ if non-linear elastic elements are used, as shown in Fig. 1. Fig. 8 shows the simulation result for no damping on the joints, using the metric $M(q)$. In case of damping on the joints, similar, but damped, responses were observed which are analytically described through $M_{q}=B$.

The two identical non-linear elastic elements were modeled by $F_{\ell}=k \cdot \ell^{2}$, with $k=100 \mathrm{~N} / \mathrm{m}$ and sufficient pretension $\left(z_{1}=z_{2}=1 \mathrm{~m}\right)$ to prevent $\ell \leq 0 \mathrm{~m}$. The input positions $z_{1}, z_{2}$ are driven in common mode and change in two smooth steps from 1 to 3 to $5 \mathrm{~m}$.

Fig. 8 shows that the frequency changes after each input change, which confirms the variation of the compliance.

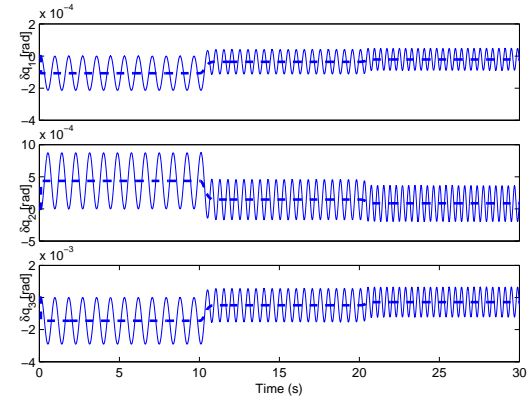

Fig. 8. Changing position $z$ alters compliance (No damping, $\delta^{c} W_{e}=$ $(0,0,0,0.0001 N, 0,0))$. Infinitesimal joint displacement $\delta q$ : simulated response [solid line] vs. analytically determined response [dashed line], using metric: $M_{q}=M(q)$.

Furthermore, it is confirmed that also for changing input positions, the analytically determined $\delta q_{e}$ indeed corresponds to the simulated response.

\section{CONClusions And Future Work}

This paper presented the analysis of the finger-tip compliance of an under-actuated robotic finger, based on geometric decomposition of the configuration space into a subspace of null-space motions (infinite compliance) and its reciprocal space of finite compliance displacements. For the decomposition a physical meaningful metric was found for two separate cases: dynamics with and without damping. The compliance analysis was confirmed by simulation results for both cases. Finally, the variability property of the compliance was confirmed by simulation and shown to be in accordance with the theoretical results. Additionally, a design trade off was formulated to optimize the robotic design for external wrenches which need to be altered with finite compliance.

In future work, the authors plan to validate the presented results on a test-setup. Furthermore, the theoretical results will be integrated into the design of an impedance controller.

\section{REFERENCES}

[1] A. Bicchi, "Hands for dexterous manipulation and robust grasping: a difficult road toward simplicity," IEEE Trans. on Rob. and Autom. vol. 16, no. 6, pp. 652-662, Dec. 2000.

[2] B. Massa, S. Roccella, M. C. Carrozza, and P. Dario, "Design and development of an underactuated prosthetic hand," in IEEE Int. Conf. on Rob. and Autom., vol. 4, May 2002.

[3] M. Wassink, R. Carloni, and S. Stramigioli, "Port-hamiltonian analysis of a novel robotic finger concept for minimal actuation variable impedance grasping," in IEEE Int. Conf. on Rob. and Autom., May 2010.

[4] S. Hirose and Y. Umetani, "The development of soft gripper for the versatile robot hand," Mechanism and Machine Theory, vol. 13, pp. 351-359, 1978.

[5] L. Birglen, T. Laliberte, and C. Gosselin, Underactuated Robotic Hands, ser. Springer Tracts in Advanced Robotics. Springer-Verlag Berlin Heidelberg, 2008.

[6] S. Stramigioli, Modeling and IPC Control of Interactive Mechanical Systems, A Coordinate-Free Approach, 1st ed. Springer-Verlag, 2001.

[7] T. Pigoski, M. Griffis, and J. Duffy, "Stiffness mappings employing different frames of reference," Mech. Mach. Theory, vol. 33, no. 6, pp. 825-838, 1998.

[8] K. L. Doty, C. Melchiorri, C. Bonivento, and E. M. Schwartz, "Robo manipulability," IEEE Trans. on Rob and Autom., vol. 11, no. 3, 1995.

[9] K. L. Doty, C. Melchiorri, and C. Bonivento, "A theory of generalized inverses applied to robotics," Int. J. of Rob. Res., vol. 12, no. 1, 1993. 